\title{
Qualidade seminal e perfil metabólico de caprinos alimentados com semente de linhaça na dieta
}

[Semen quality and metabolic profile of goats fed flaxseed in the diet]

\author{
R.S. Souza ${ }^{l}$, L.P. Barbosa ${ }^{1 *}$, A.M. Pinheiro ${ }^{1}$, W.M. Machado ${ }^{2}$, \\ C.S. Mendes ${ }^{1}$, M.L. Araujo ${ }^{3}$, D.O. Souza ${ }^{1}$, A.L.A. Santana ${ }^{4}$ \\ ${ }^{1}$ Centro de Ciências Agrárias, Ambientais e Biológicas - UFRB - Cruz das Almas, BA \\ ${ }^{2}$ Aluno de pós-graduação - Departamento de Ciências Agrárias e Ambientais - UESC - Ilhéus, BA \\ ${ }^{3}$ Aluno de pós-graduação - Escola de Medicina Veterinária e Zootecnia - UFBA - Salvador, BA \\ ${ }^{4}$ Instituto de Ciências Agrárias - UFVJM - Unaí, MG
}

\begin{abstract}
RESUMO
O objetivo da presente pesquisa foi avaliar o efeito da inclusão de semente de linhaça na dieta sobre a qualidade seminal e o perfil metabólico de machos caprinos. Foram utilizados 16 machos da raça Saanen, distribuídos aleatoriamente em quatro grupos, recebendo níveis de inclusão de semente de linhaça $(0,4,8$ e 12\%) na dieta, durante um período de 60 dias. As coletas e as avaliações seminais foram realizadas duas vezes por semana. Os animais foram submetidos a coletas quinzenais de sangue, durante todo o período experimental. Os dados foram avaliados por ANOVA e pela análise de regressão a $5 \%$ de significância. Houve comportamento cúbico para motilidade espermática progressiva, que apresentou o maior valor $(82,30 \%)$ com a adição de $9,92 \%$ de semente de linhaça na dieta $(\mathrm{P}<0,05)$. Houve comportamento cúbico para concentração plasmática de triglicerídeos, e o nível de $8 \%$ de semente de linhaça na dieta apresentou média de $43,32 \mathrm{mg} \mathrm{dL}^{-1}$, acima dos valores normais para a espécie caprina $(\mathrm{P}<0,05)$. A inclusão de semente de linhaça na dieta de machos caprinos proporcionou melhora na produção espermática e na qualidade seminal. No entanto, devem-se considerar os possíveis efeitos dos níveis superiores a 4\% de semente de linhaça sobre o perfil metabólico dos animais.
\end{abstract}

Palavras-chave: ácido alfalinolênico, perfil lipídico, reprodução

\begin{abstract}
This study aimed to evaluate the effect of flaxseed inclusion in the diet of male goats through the semen quality and metabolic profile. Randomly allocated 16 Saanen males were placed into four groups receiving flaxseed inclusion levels $(0,4,8$ and $12 \%)$ in the diet over a period of 60 days. The collections and seminal evaluations were performed twice a week. The animals were subjected to biweekly blood collections from the beginning to the end of the trial period. The data were evaluated by ANOVA and regression analysis at 5\% significance. A cubic behavior for motility, with the highest value (82.30\%) with the addition of $9.92 \%$ of flaxseed in the diet $(P<0.05)$ was detected. A cubic behavior for plasma triglyceride concentration and the level of $8 \%$ of flaxseed in the diet averaged $43.32 \mathrm{mg}^{d-1}$ above normal values for goats $(P<0.05)$ was detected. The inclusion of flaxseed in the diet of male goats improved sperm production and quality. However, one should consider the possible effects of the levels above $4 \%$ of flaxseed on the metabolic profile of the animals.
\end{abstract}

Keywords: alpha-linolenic acid, lipid profile, reproduction

\section{INTRODUÇÃO}

A reprodução tem suas funções comprometidas em situações de desequilíbrio nutricional, as quais são primariamente resultantes de falhas no ajuste do balanço entre a disponibilidade de

Recebido em 23 de agosto de 2017

Aceito em 17 de setembro de 2018

*Autor para correspondência (corresponding author)

E-mail: larissa@ufrb.edu.br nutrientes e seus requerimentos, tanto pelos animais em reprodução como por aqueles que irão ainda iniciar sua vida reprodutiva (Almeida et al., 2007). Nos reprodutores caprinos, por exemplo, alterações na nutrição podem levar a respostas no tamanho dos testículos e, consequentemente, na produção de espermatozoides (Eloy e Pereira, 2013). 
A suplementação lipídica, com o uso de óleos, é uma alternativa para fornecer rações com maior densidade aos animais (Paula et al., 2012). Diferentes tipos de lipídeos têm sido fornecidos a animais de produção com o objetivo de melhorar os processos reprodutivos, incluindo os lipídeos de graxarias, as granuladas e as provenientes de sementes de oleaginosas (Staples et al., 2009). Entre as fontes vegetais mais utilizadas para tal fim, estão incluídas a semente de canola, a semente de linhaça (Staples et al., 2009) e a semente ou o óleo de girassol (Cordeiro et al., 2015).

A semente de linhaça é conhecida por conter elevado teor de lipídeos (33 a 34\%), sendo mais de $50 \%$ desses do ácido graxo insaturado $\alpha$ linolênico (18:3n-3), pertencente à série ômega-3 $(\omega-3)$, e, em média, $13,35 \%$ de ácido linoleico (18:2n-6) da série ômega-6 ( $\omega-6)$, além de ácidos graxos monoinsaturados, saturados, vitaminas e compostos fenólicos (Barroso et al., 2014).

Assim, o objetivo deste trabalho foi avaliar o efeito da inclusão de semente de linhaça na dieta sobre a qualidade seminal e o perfil metabólico de machos caprinos.

\section{MATERIAL E MÉTODOS}

O estudo foi executado na Estação Experimental Cruzeiro do Mocó, pertencente à Empresa
Baiana de Desenvolvimento Agrícola (EBDA), localizada na cidade de Feira de Santana-BA (12 ${ }^{\circ}$ $16^{\prime} 00^{\prime \prime} \mathrm{S}$ e $\left.38^{\circ} 58^{\prime} 00^{\prime \prime} \mathrm{W}\right)$, situada a 234 metros de altitude acima do nível do mar, com temperatura média de $26,5^{\circ} \mathrm{C}$ e índices pluviais médios variando de 900 a $1.200 \mathrm{~mm}$ anuais (Estação Climatológica da Universidade Estadual de Feira de Santana 83221-Inmet/DTEC).

Foram utilizados 16 caprinos adultos da raça Saanen, com idade de $2,12 \pm 084$ e peso médio de $39,34 \pm 16,72 \mathrm{~kg}$. Os animais foram manejados em baias coletivas de $16 \mathrm{~m}^{2}$ suspensas, com piso ripado de madeira e cobertura de telhas de fibrocimento, providas de bebedouros e comedouros.

Os animais foram distribuídos aleatoriamente em quatro grupos experimentais, nos quais receberam 0 (2,12 $\pm 0,85$ anos), $4(1,87 \pm 0,85$ anos), 8 (2,37 $\pm 1,10$ anos), e $12 \% \quad(2,12 \pm 0,85$ anos) de semente de linhaça, com base na matéria seca das dietas, durante um período de 60 dias. As dietas foram formuladas segundo o NRC (Nutrient..., 2007) (Tab. 1) e fornecidas duas vezes ao dia, de maneira a proporcionar sobras entre 10 e $20 \%$, mantendo a proporção volumoso:concentrado de 60:40, sob a forma de mistura completa. $\mathrm{O}$ volumoso fornecido foi feno de Tifton-85 (Cynodon sp.), moído em partícula de aproximadamente $5 \mathrm{~cm}$. A água foi fornecida ad libitum.

Tabela 1. Proporção dos ingredientes das dietas

\begin{tabular}{lcccc}
\hline \multirow{2}{*}{ Ingredientes (\%MS) } & \multicolumn{4}{c}{ Semente de linhaça (\% MS) } \\
\cline { 2 - 5 } & 0 & 4 & 8 & 12 \\
\hline Semente de linhaça & 0,00 & 4,00 & 8,00 & 12,00 \\
Premix vitamínico mineral $^{1}$ & 1,20 & 1,20 & 1,20 & 1,20 \\
Farelo de soja $_{\text {Farelo de milho }}$ & 11,20 & 9,30 & 7,40 & 5,52 \\
Ureia & 27,20 & 25,10 & 23,00 & 20,88 \\
Feno & 0,40 & 0,40 & 0,40 & 0,40 \\
Total & 60,00 & 60,00 & 60,00 & 60,00 \\
\hline
\end{tabular}

${ }^{1}$ Níveis de garantia (por kg em elementos ativos): cálcio 120,00g; fósforo 87,00g; sódio 147,00g; enxofre 18,00g; cobre 590,00mg; cobalto 40,00mg; cromo $20,00 \mathrm{mg}$; ferro $1.800,00 \mathrm{mg}$; iodo $80,00 \mathrm{mg}$; manganês $1.300,00 \mathrm{mg}$; selênio 15,00mg; zinco 3.800,00mg; molibdênio 300,00mg; flúor máximo 870,00mg; solubilidade do fósforo (P) em ácido cítrico a $2 \%$ mínimo - $95 \%$.

A composição bromatológica das dietas experimentais (Tab. 2) foi determinada conforme a Association of Official Analytical Chemists AOAC (Official..., 1990), para determinação dos teores de matéria seca (MS), matéria mineral $(\mathrm{MM})$, proteína bruta $(\mathrm{PB})$ e extrato etéreo $(\mathrm{EE})$.
As análises para a determinação da fibra em detergente neutro (FDN), fibra em detergente ácido (FDA), celulose, hemicelulose e lignina foram realizadas segundo Van Soest et al. (1991). Os carboidratos não fibrosos foram calculados de acordo com Sniffen et al. (1992). 
Qualidade seminal...

Tabela 2. Composição bromatológica das dietas experimentais

\begin{tabular}{lccccc}
\multicolumn{1}{c}{ Nutrientes (\% matéria seca) } & \multicolumn{4}{c}{ Nível de linhaça (\%) } & \multirow{2}{*}{ Feno } \\
\cline { 2 - 5 } & 0 & 4 & 8 & 12 & \\
\hline Matéria seca & 100,00 & 100,00 & 100,00 & 100,00 & 89,47 \\
Matéria mineral & 4,82 & 4,39 & 4,52 & 4,21 & 4,27 \\
Proteína bruta & 24,59 & 24,43 & 22,35 & 23,36 & 2,17 \\
Extrato etéreo & 2,16 & 6,07 & 9,87 & 12,93 & 0,71 \\
Fibra em detergente neutro & 32,71 & 31,28 & 28,24 & 47,22 & 76,96 \\
Fibra em detergente ácido & 6,65 & 8,10 & 8,49 & 10,97 & 41,32 \\
Lignina & 1,58 & 1,71 & 2,35 & 3,07 & 8,56 \\
Celulose & 5,07 & 6,39 & 6,14 & 7,90 & 32,76 \\
Hemicelulose & 26,06 & 23,18 & 19,74 & 36,25 & 35,64 \\
Carboidratos não fibrosos & 35,71 & 33,83 & 35,02 & 12,28 & 15,88 \\
\hline
\end{tabular}

Para avaliação da qualidade física e morfológica seminal, as coletas de sêmen iniciaram 25 dias após o início do fornecimento das dietas, pela técnica de vagina artificial, utilizando-se uma fêmea em estro como manequim, duas vezes por semana, totalizando 10 coletas por animal. Após a coleta, os ejaculados foram acondicionados em banho-maria a $37^{\circ} \mathrm{C}$ e submetidos ao exame dos aspectos físicos e morfológicos seminais, segundo o CBRA (Henry et al., 2013). Para avaliação da morfologia espermática, foi utilizada a técnica de lâmina úmida em microscopia de interferência diferencial de fase.

Foram realizadas coletas de sangue no primeiro dia do experimento, antes da administração das dietas. Posteriormente, os animais foram submetidos a coletas quinzenais, até o fim do período experimental, totalizando cinco coletas. As coletas foram realizadas por venopunção da jugular, utilizando-se tubos de coleta a vácuo com anticoagulante ácido etilenodiamino tetraacético (concentração de k3EDTA + gel separador) de $4,5 \mathrm{~mL}$ (Vacuette ${ }^{\circledR}$, Shandong Weigao Medical Group Polymer, Weihai, China). Para determinação da concentração de glicose, foram utilizados tubos de coleta a vácuo contendo solução anticoagulante (concentração de k3EDTA e o estabilizador fluoreto de sódio) de 4,0mL (Vacuette ${ }^{\circledR}$, Greiner Bio-One, Áustria). Após as coletas, o sangue foi centrifugado a 3000rpm por 15 minutos, e o plasma sanguíneo acondicionado em microtubos de polietileno a $20^{\circ} \mathrm{C}$.
A concentração plasmática de glicose, colesterol total, colesterol-HDL, triglicerídeos e ureia foi determinada por meio de análise colorimétrica, em triplicatas, utilizando-se kits comerciais (Doles ${ }^{\circledR}$, Ltda., Goiânia, GO, Brasil), conforme especificações do fabricante. Para obtenção das concentrações de colesterol-VLDL e do colesterol-LDL, empregaram-se as equações de Friedewald et al. (1972):

colesterol-VLDL $=($ triglicerídeos $/ 5)$; colesterol-LDL $=$ [colesterol total - (colesterolHDL + colesterol-VLDL)].

Para determinação da concentração plasmática de testosterona, o sangue com anticoagulante (com concentração de k3EDTA e o estabilizador fluoreto de sódio) foi centrifugado a 3000rpm por 15 minutos. Retiraram-se alíquotas de $1,0 \mathrm{~mL}$ de plasma sanguíneo, as quais foram acondicionadas em microtubos de polietileno a $20^{\circ} \mathrm{C}$, que foram encaminhados para o Laboratório de Reprodução Animal do Departamento de Zootecnia da Universidade Federal de Viçosa-MG, onde se procedeu à realização da análise pelo método de quimioluminescência, utilizando-se o kit comercial (Access Testosterona, Beckman Coulte ${ }^{\circledR}$, Pasadena, CA, USA), seguindo as orientações recomendadas pelo fabricante.

A normalidade dos dados foi verificada pelo teste de Shapiro-Willk. Os dados foram submetidos à análise de variância e ao teste de regressão, adotando-se o nível de $5 \%$ de 
significância, por meio da utilização do software estatístico de análise preditiva (SPSS 13.0).

O projeto foi executado de acordo com os regulamentos aprovados pelo Comitê de Ética no Uso de Animais da UFRB (Protocolo $\mathrm{n}^{\circ}$ 23007.006635/2014-60).

\section{RESULTADOS E DISCUSSÃO}

A inclusão de semente de linhaça na dieta de caprinos não alterou $o$ aspecto seminal (coloração e consistência seminal), com predominância da coloração marfim e consistência leitosa $(\mathrm{P}>0,05)$. Os parâmetros encontrados estão de acordo com o preconizado pelo CBRA (Henry et al., 2013). Houve diferença significativa $(\mathrm{P}<0,05 ; \hat{\mathrm{y}}=0,7055 \quad-$ $\left.0,0549 x+0,0073 x^{2} ; R 2=0,917\right)$ para volume seminal, que apresentou comportamento quadrático positivo. O grupo com inclusão de $12 \%$ de semente de linhaça na dieta apresentou volume superior aos demais, com valores entre $0,68 \pm 0,46 \mathrm{~mL}(\mathrm{G} 0 \%)$ e $1,13 \pm 0,57 \mathrm{~mL}(\mathrm{G} 12 \%)$ (Tab. 3). Os valores encontrados estão de acordo com o preconizado pelo CBRA (Henry et al., 2013), tendo como valor médio $0,8 \mathrm{~mL}$.

Não houve diferença significativa $(\mathrm{P}>0,05)$ para turbilhonamento espermático, com valores entre

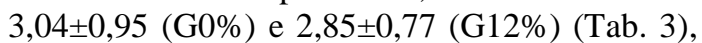
e esses resultados encontram-se dentro do preconizado pelo CBRA (Henry et al., 2013) para sêmen in natura da espécie caprina.

Tabela 3. Aspectos físicos do sêmen in natura de machos caprinos suplementados com níveis de semente de linhaça na dieta

\begin{tabular}{lcccc}
\multicolumn{1}{c}{ Parâmetros } & \multicolumn{3}{c}{ Níveis de semente de linhaça (\%) } \\
\cline { 2 - 5 } & 0 & 4 & 8 & 12 \\
\hline Volume seminal (mL)* & $0,68 \pm 0,46^{\mathrm{b}}$ & $0,68 \pm 0,32^{\mathrm{b}}$ & $0,66 \pm 0,24^{\mathrm{b}}$ & $1,13 \pm 0,57^{\mathrm{a}}$ \\
Turbilhonamento espermático & $3,04 \pm 0,95$ & $2,63 \pm 1,48$ & $3,15 \pm 0,99$ & $2,85 \pm 0,77$ \\
$(0-5)^{\mathrm{ns}}$ & & & & \\
Motilidade espermática $_{\text {progressiva }(0-100 \%)^{*}}$ & $76,40 \pm 16,75^{\mathrm{b}}$ & $66,39 \pm 28,88^{\mathrm{b}}$ & $79,17 \pm 18,27^{\mathrm{a}}$ & $77,00 \pm 14,79^{\mathrm{b}}$ \\
Vigor espermático (0-5)* & $4,47 \pm 0,71^{\mathrm{b}}$ & $3,94 \pm 1,61^{\mathrm{b}}$ & $4,58 \pm 0,87^{\mathrm{b}}$ & $4,64 \pm 0,54^{\mathrm{a}}$ \\
Concentração espermática & $1,83 \pm 1,37^{\mathrm{b}}$ & $1,69 \pm 1,34^{\mathrm{b}}$ & $2,16 \pm 1,28^{\mathrm{b}}$ & $5,20 \pm 3,28^{\mathrm{a}}$ \\
(x10 $^{9}$ sptz/mL)* & &
\end{tabular}

Os valores descritos no corpo da Tabela referem-se à média \pm desvio-padrão; * = significativo a 0,05 de probabilidade; ns = não significativo. Valores com letras minúsculas diferentes, sobrescritas na mesma linha, diferem significativamente $(\mathrm{a}-\mathrm{b}, \mathrm{P}<0,05)$.

Houve comportamento cúbico para motilidade espermática progressiva $(\mathrm{P}<0,05 ; \hat{\mathrm{y}}=76,4 \quad-$ $\left.8,4962 x+1,8916 x^{2}-0,0983 x^{3} ; R 2=1,0\right)$ (Fig. 1), que apresentou uma derivação do maior valor $(82,30 \%)$ com um nível de adição de linhaça na dieta de $9,92 \%$ (Tab. 3). Esses resultados foram semelhantes aos encontrados por Dolatpanah et al. (2008), quando trabalharam com óleo de peixe e vitamina E na dieta de caprinos, os quais obtiveram $83,68 \%$ para motilidade espermática. Gonçalves et al. (2014), ao trabalharem com a inclusão de ácidos graxos poli-insaturados oriundos do óleo de palma na dieta, a $2 \%$ da matéria seca total, não obtiveram influência na motilidade espermática do sêmen de búfalos, mas o efeito foi positivo na habilidade de progressão das células espermáticas após 90 dias do início da suplementação, estando essa característica relacionada ao aumento do potencial de fertilidade do sêmen após a descongelação.

Moallem et al. (2015) avaliaram as características seminais de touros suplementados com de óleo de linhaça ou óleo de peixe na dieta, por um período de 13 semanas, e verificaram que houve uma mudança moderada de ácido docosahexaenoico (DHA) e ácido docosapentaenoico (DPA) no sêmen do grupo suplementado com óleo de linhaça, melhorando a sobrevivência e a qualidade do sêmen fresco ou congelado-descongelado, o que sugere que a razão entre esses dois ácidos graxos desempenha um papel importante na função espermática. Samadian et al. (2010), em estudos com inclusão de PUFAs n-3 na dieta de ovinos, obtiveram um aumento significativo da concentração de DHA nos espermatozoides, acompanhado por melhoria da qualidade do sêmen. 


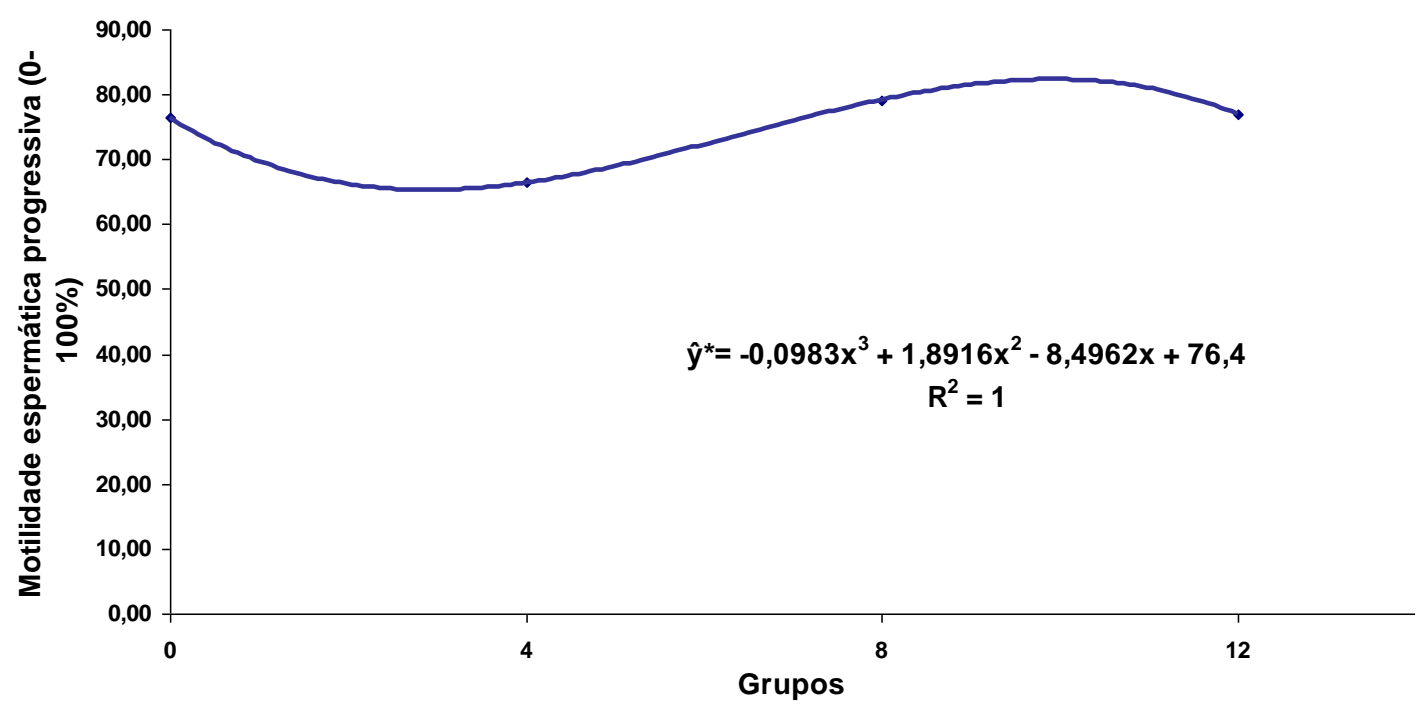

Figura 1. Motilidade espermática progressiva (0-100\%) de caprinos suplementados com 0, 4, 8 e $12 \%$ de semente de linhaça na dieta. Os dados foram avaliados por meio da análise de regressão.

Houve comportamento quadrático positivo para vigor espermático $(\mathrm{P}<0,05 ; \hat{\mathrm{y}}=4,3825-0,0819 \mathrm{x}$ $\left.+0,0092 x^{2} ; R 2=0,5\right)($ Tab. 3), por meio do qual se avalia o tipo de movimento que os espermatozoides de uma amostra apresentam, e, no presente experimento, observou-se que a inclusão de níveis da semente de linhaça na dieta dos caprinos promoveu melhora no vigor das células espermáticas, possivelmente pela incorporação dos ácidos graxos poli-insaturados da dieta e pelas modificações estruturais nessas células, pois, de acordo com Lottenberg (2009), é possível ocorrer alterações estruturais e funcionais na membrana fosfolipídica das células por meio da ingestão de ácido graxo ômega-3, e tais modificações permitem maior fluidez da membrana celular e, consequentemente, maior mobilidade das proteínas, o que favorece a troca de sinais de transdução, interação hormônioreceptor e transporte de substratos entre os meios intra e extracelular.

Houve diferença significativa entre os grupos para concentração espermática, que apresentou comportamento quadrático positivo $(\mathrm{P}<0,05$; $\left.\hat{y}=1,9352-0,3317 x+0,0497 x^{2} ; R 2=0,9772\right)$, também observado pela inclusão da semente de linhaça na dieta dos caprinos (Tab. 3). Resultado similar foi descrito por Dolatpanah et al. (2008), ao suplementarem caprinos com 2,5\% de óleo de peixe, fonte de ômega 3 e vitamina $E$, os quais verificaram melhora significativa na concentração de espermatozoides. $\mathrm{O}$ mesmo estudo sugere um efeito favorável da suplementação com óleo de peixe sobre a qualidade do sêmen, devido ao aumento das concentrações de eicosanoides (prostaglandinas, prostaciclinas, tromboxanos e leucotrienos), que podem ser relevantes no controle hormonal da espermatogênese.

Os resultados de morfologia espermática estão descritos na Tab. 4. Houve comportamento quadrático negativo para defeitos maiores $\left(\mathrm{P}<0,05 ; \quad \hat{\mathrm{y}}=3,2758+0,8279 \mathrm{x}-0,0606 \mathrm{x}^{2}\right.$; $\mathrm{R} 2=0,9802)$, para defeitos menores $(\mathrm{P}<0,05$; $\left.\hat{y}=7,9998+0,4259 x-0,0628 x^{2} ; R 2=0,9628\right) e$ para defeitos totais $\left(\mathrm{P}<0,05 ; \hat{\mathrm{y}}^{* *}=11,394+\right.$ $\left.1,2474 x-0,1238 x^{2} ; R 2=0,9581\right)$. 
Tabela 4. Aspectos morfológicos do sêmen in natura de machos caprinos suplementados com níveis de semente de linhaça na dieta

\begin{tabular}{|c|c|c|c|c|}
\hline & \multicolumn{4}{|c|}{ Níveis de semente de linhaça } \\
\hline & 0 & 4 & 8 & 12 \\
\hline Defeitos maiores $(0-100 \%)^{*}$ & $3,34 \pm 3,42^{\mathrm{a}}$ & $5,41 \pm 5,02^{b}$ & $6,23 \pm 4,06^{b}$ & $4,42 \pm 3,20^{\mathrm{a}}$ \\
\hline Defeitos menores $(0-100 \%)^{*}$ & $8,16 \pm 6,3^{\mathrm{b}}$ & $8,23 \pm 6,32^{b}$ & $7,85 \pm 6,08^{b}$ & $3,91 \pm 2,98^{\mathrm{a}}$ \\
\hline Defeitos totais $(0-100 \%) *$ & $11,60 \pm 8,14^{\mathrm{b}}$ & $13,77 \pm 8,94^{\mathrm{b}}$ & $14,08 \pm 6,61^{\mathrm{b}}$ & $8,32 \pm 5,08^{\mathrm{a}}$ \\
\hline
\end{tabular}

Os valores descritos no corpo da Tabela referem-se à média \pm desvio-padrão; * = significativo a 0,05 de probabilidade. Valores com letras minúsculas diferentes, sobrescritas na mesma linha, diferem significativamente $(\mathrm{a}-\mathrm{b}, \mathrm{P}<0,05)$.

Os resultados obtidos para os parâmetros de morfologia seminal estão dentro dos valores padrão preconizados pelo CBRA (Henry et al., 2013). Obteve-se, no presente estudo, redução das alterações de cauda enrolada e dobrada (defeitos menores) e de cauda fortemente enrolada e peça intermediária irregular (defeitos maiores). A adição de $12 \%$ de semente de linhaça melhorou a eficiência da espermatogênese por diminuir a ocorrência de patologias espermáticas nos animais, o que se deve possivelmente à incorporação dos ácidos graxos poli-insaturados da linhaça à membrana plasmática dos espermatozoides.

Os lipídeos desempenham papel fundamental na estrutura e na função das células dos mamíferos (Dunning et al., 2014), sendo os ácidos graxos poli-insaturados componentes da membrana plasmática espermática, especialmente o DHA, componente essencial dos espermatozoides saudáveis, pois aumenta a integridade da membrana e a flexibilidade da cauda (Brinsko et al., 2005) e está correlacionado com o aumento da concentração e da motilidade espermática (Samadian et al., 2010).

Como a linhaça é uma excelente fonte de ácidos graxos poli-insaturados, principalmente na forma de ácido alfalinolênico (ômega-3) (Barroso et al., 2014), o qual é convertido em DHA, sugere-se que a adição de tais componentes na dieta pode ter sido incorporada à membrana plasmática espermática, resultando em melhor qualidade dessa célula, observada pela redução dos defeitos menores e, consequentemente, os totais, cujo valor obtido está abaixo do máximo aceitável (20\%) pelo CBRA (Henry et al., 2013).

A inclusão de semente de linhaça na dieta de machos caprinos alterou o perfil metabólico dos animais (Tab. 5). A concentração plasmática de glicose apresentou comportamento linear decrescente $\left(\mathrm{P}<0,05 ; \hat{\mathrm{y}}^{* *}=57,022\right.$ - 1,2189x; $\mathrm{R} 2=0,9929)$. Apesar de Araújo e Silva (2008) considerarem que a concentração plasmática de glicose entre 45,2 - 51,5mg $\mathrm{dL}^{-1}$ deve ser utilizada como referência para se chegar a um diagnóstico envolvendo problemas no metabolismo e/ou na alimentação dos animais, González e Silva (2006) ressaltam que, na digestão dos ruminantes, pouca glicose proveniente da dieta entra na corrente sanguínea, pois o fígado é o órgão responsável pela sua síntese por meio de moléculas precursoras na via da gliconeogênese.

De acordo com Huang et al. (2009), alto nível de lipídeo na alimentação de ruminantes pode reduzir a digestão da fibra no rúmen, provocando, consequentemente, menor disponibilidade de energia, o que pode ter ocorrido no presente estudo, pois à medida que se aumentou o nível de inclusão de linhaça, obteve-se, na composição da dieta, aumento nos níveis de extrato etéreo e fibra, e redução dos carboidratos não fibrosos (Tab. 2). Tal composição pode ter influenciado na concentração de glicose sanguínea. Entretanto, é válido ressaltar que os níveis de testosterona não foram influenciados pela dieta. 
Qualidade seminal...

Tabela 5. Perfil metabólico de caprinos suplementados com níveis de semente de linhaça na dieta

\begin{tabular}{lcccc}
\hline \multirow{2}{*}{ Parâmetros $\left(\mathrm{mg} \mathrm{dL}^{-1}\right)$} & \multicolumn{4}{c}{ Níveis de semente de linhaça (\%) } \\
\cline { 2 - 5 } & 0 & 4 & 8 & 12 \\
\hline Glicose* $^{*}$ & $56,61 \pm 7,48^{\mathrm{a}}$ & $51,84 \pm 11,39^{\mathrm{b}}$ & $44,25 \pm 17,72^{\mathrm{b}}$ & $38,83 \pm 14,87^{\mathrm{b}}$ \\
Triglicerídeos* $^{\mathrm{a}}$ & $22,44 \pm 6,79^{\mathrm{b}}$ & $16,75 \pm 10,04^{\mathrm{b}}$ & $43,32 \pm 51,97^{\mathrm{a}}$ & $18,61 \pm 11,53^{\mathrm{b}}$ \\
Colesterol total $^{\mathrm{n}}$ & $45,89 \pm 17,13$ & $45,94 \pm 15,54$ & $72,25 \pm 46,24$ & $48,45 \pm 42,85$ \\
HDL $^{* *}$ & $17,95 \pm 13,89^{\mathrm{b}}$ & $19,03 \pm 11,31^{\mathrm{b}}$ & $43,01 \pm 15,64^{\mathrm{a}}$ & $33,52 \pm 14,48^{\mathrm{b}}$ \\
LDL $^{\mathrm{ns}}$ & $23,45 \pm 11,76$ & $23,55 \pm 9,27$ & $20,56 \pm 53,41$ & $11,21 \pm 40,25$ \\
VLDL $^{* *}$ & $4,48 \pm 1,36^{\mathrm{b}}$ & $3,35 \pm 2,01^{\mathrm{b}}$ & $8,66 \pm 10,39^{\mathrm{a}}$ & $3,72 \pm 2,53^{\mathrm{b}}$ \\
Ureia $^{\text {ns }}$ & $34,93 \pm 14,35$ & $52,24 \pm 29,85$ & $48,04 \pm 39,32$ & $44,77 \pm 6,72$ \\
Testosterona $\left(\mathrm{x} 10^{-6}\right)^{\mathrm{ns}}$ & $2,99 \pm 3,33$ & $3,58 \pm 4,12$ & $2,82 \pm 3,31$ & $1,76 \pm 1,14$ \\
\hline
\end{tabular}

HDL= lipoproteína de alta densidade; LDL= lipoproteína de baixa densidade; VLDL= lipoproteína de muito baixa densidade. Os valores descritos no corpo da Tabela referem-se à média \pm desvio-padrão; * = significativo a 0,05 de probabilidade; ${ }^{\mathrm{ns}}=$ não significativo. Valores com letras minúsculas diferentes, sobrescritas na mesma linha, diferem significativamente $(a-b, P<0,05)$.

Houve comportamento cúbico para concentração de triglicerídeos no plasma dos caprinos $(\mathrm{P}<0,05$; $\hat{y}=22,44-12,417 x+3,6187 x^{2}-0,2176 x^{3} ;$ $\mathrm{R} 2=1,0$ ) (Fig. 2), que apresentou derivação com um nível máximo de $8,96 \%$ de semente de linhaça para obter uma média estimada de $45,17 \mathrm{mg} \quad \mathrm{dL}^{-1}$ (Tab. 5). A concentração plasmática de triglicerídeos obtida neste estudo para o nível de $8 \%$ de semente de linhaça na dieta encontra-se acima do relatado por Moraes et al. (2014), os quais incluíram 9,5\% de semente de linhaça na dieta de caprinos e obtiveram $32,48 \mathrm{mg} \mathrm{dL}^{-1}$, valor superior ao obtido nos animais do grupo controle. De acordo com esses autores, tal efeito é compatível com dietas com níveis elevados de gordura.

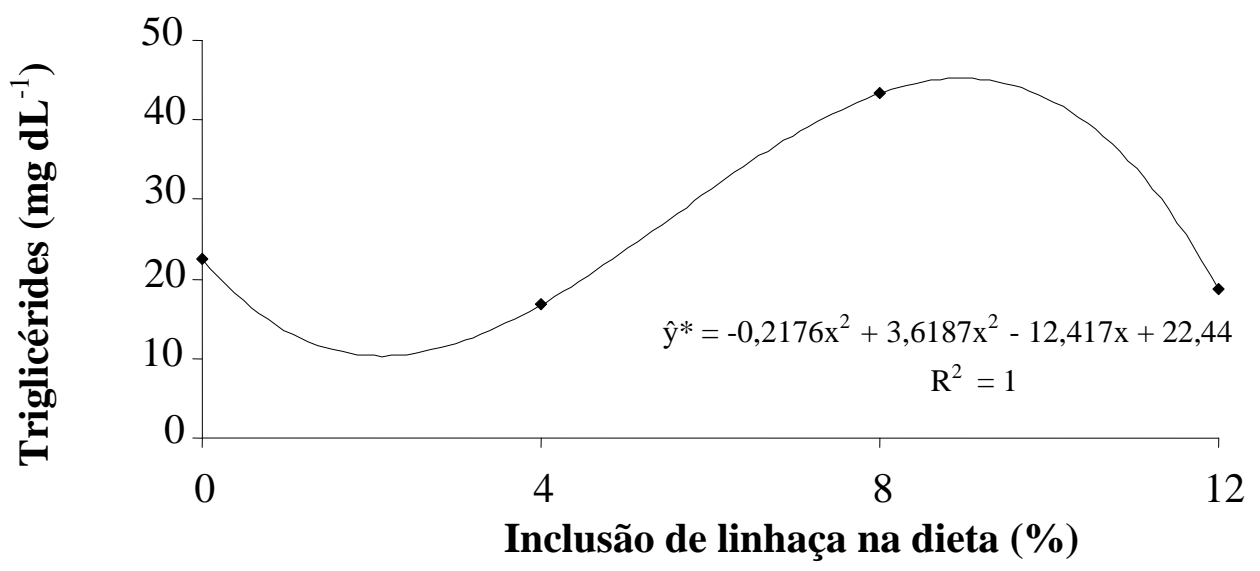

Figura 2. Concentração plasmática de triglicerídeos $\left(\mathrm{mg} \mathrm{dL}^{-1}\right)$ de caprinos suplementados com $0,4,8$ e $12 \%$ de semente de linhaça na dieta. Os dados foram avaliados por meio de análise de regressão.

Não houve diferença significativa para concentração plasmática de colesterol total entre os grupos experimentais, com valores entre $45,89 \pm 17,13 \mathrm{mg} \mathrm{dL} \mathrm{L}^{-1}(\mathrm{G} 0 \%)$ e $72,25 \pm 46,24 \mathrm{mg}$ $\mathrm{dL}^{-1}(\mathrm{G} 8 \%)(\mathrm{P}>0,05)$ (Tab. 5). Esse resultado mostra-se semelhante ao de Moraes et al. (2014), que obtiveram $72,30 \mathrm{mg} \mathrm{dL}^{-1}$ de colesterol total em caprinos alimentados com dieta suplementada com 9,5\% de semente de linhaça, e não obtiveram diferença entre o grupo tratado e o grupo controle.
A inclusão de semente de linhaça na dieta dos caprinos resultou em comportamento cúbico para concentração plasmática de HDL $(\mathrm{P}<0,05$; $\left.\hat{y}=17,95-7,29 x+2,4772 x^{2}-0,1468 x^{3} ; R 2=1,0\right)$, que apresentou nível máximo ótimo de $9,50 \%$ para obter concentração estimada de 46,39mg $\mathrm{dL}^{-1}$, mas não promoveu diferença na concentração plasmática de LDL ( $\mathrm{P}>0,05)$ (Tab. 5). Segundo Lottenberg (2009), os ácidos graxos poli-insaturados modulam diversos genes envolvidos nos processos oxidativos, inclusive 
bloqueando aqueles relacionados com a lipogênese. $\mathrm{O}$ mesmo autor relata, em seu trabalho, que a suplementação de EPA/DHA pode aumentar discretamente os níveis de HDL e reduzir os de LDL, e tal efeito pode estar associado aos ácidos linolênico ou linoleico e outros constituintes não proteicos presentes nessas sementes.

Os resultados do presente estudo estão de acordo com Beynen et al. (2000), que encontraram diferença significativa para concentração sérica de HDL ao utilizarem diferentes quantidades e tipos de lipídeos na dieta de caprinos. Os mesmos autores observaram que as dietas com alto teor de lipídeos, quando comparada com as dietas com baixo teor de lipídeos, aumentaram significativamente a concentração plasmática de colesterol total, HDL, triglicerídeos e fosfolipídeos.

Houve comportamento cúbico para concentração de VLDL $\left(\mathrm{P}<0,05 ; \hat{\mathrm{y}}=4,48-2,4783 \mathrm{x}+0,7228 \mathrm{x}^{2}\right.$ $\left.0,0435 x^{3} ; R 2=1,0\right)$, que apresentou um nível máximo ótimo de $8,95 \%$ de semente de linhaça para obter uma concentração estimada de 9,01mg dL $\mathrm{m}^{-1}$ no plasma sanguíneo (Tab. 5). Diferentemente do resultado obtido no presente estudo, Soncin et al. (2009), que trabalharam com inclusão de $10 \%$ de semente de linhaça integral na dieta de equinos, não encontraram diferenças nas concentrações séricas de glicose, triglicerídeos, colesterol total, HDL e VLDL.

Não houve diferença significativa para concentração plasmática de ureia $(\mathrm{P}>0,05)$ (Tab. 5). As alterações nos níveis séricos de ureia estão correlacionadas com o teor de amônia ruminal, cuja utilização depende da atividade metabólica dos microrganismos ruminais. Neste estudo, houve uma diminuição na concentração de glicose no plasma sanguíneo dos animais alimentados com semente de linhaça. Por esse motivo, provavelmente, as concentrações plasmáticas de ureia estão acima dos valores relatados na literatura (Carvalho et al., 2010) para a espécie caprina.

Apesar de a inclusão de semente de linhaça na dieta de caprinos ter influenciado em alguns parâmetros plasmáticos, é válido ressaltar que não houve influência sobre a concentração plasmática de testosterona $(\mathrm{P}>0,05)$ (Tab. 5), o que é importante, por tratar-se de um hormônio essencial às funções reprodutivas dos machos, como espermatogênese, libido, manutenção das características sexuais secundárias, entre outras, indicando, portanto, que baixa concentração de testosterona pode influenciar de forma negativa na qualidade do sêmen e no desempenho reprodutivo do macho. Os valores obtidos no presente estudo corroboram os achados de outros pesquisadores, com valores de $1,55-2,04 \mathrm{ng} / \mathrm{mL}$ (Todini et al., 2007) e 2,5-3,0ng/mL (Talebi, 2009). Tais resultados sugerem que a suplementação da dieta com semente de linhaça não prejudica a reprodução de machos caprinos, fato observado tanto na qualidade do sêmen quanto na concentração de testosterona plasmática.

\section{CONCLUSÃO}

Os aspectos físicos e morfológicos do sêmen de caprinos apresentaram melhora à medida que se aumentou o nível de inclusão de semente de linhaça na dieta, com destaque para os níveis acima de $8 \%$ de inclusão. A inclusão de níveis de linhaça na dieta de caprinos machos alterou alguns parâmetros metabólicos desses animais, decorrente do efeito exercido pela inclusão de ácido poli-insaturado e, provavelmente, devido à composição da dieta.

\section{REFERÊNCIAS}

ALMEIDA, A.P.; SOUZA, A.L.; MENEZES, E.S.B.; ARRUDA, I.J.; RONDINA, D. Recentes avanços na relação entre nutrição e reprodução em ruminantes. In: SIMPÓSIO DE NUTRIÇÃ̃O E ALIMENTAÇÃO ANIMAL, 1., 2007, Fortaleza. Anais... Fortaleza: AMVECE, 2007. p.1-24. (Palestra).

ARAÚJO, D.F.; SILVA, I.P. Valores de amilase, glicose, colesterol e triglicérides em soro de cabras de Mossoró, RN. Acta Vet. Brasilica, v.2, p.97-100, 2008.

BARROSO, A.K.M.; TORRES, A.G.; CASTELO-BRANCO, V.N. et al. Linhaça marrom e dourada: propriedades químicas e funcionais das sementes e dos óleos prensados a frio. Ciênc. Rural, v.44, p.181-187, 2014. 
BEYNEN, A.C.; SCHONEWILLE， J.T.H.; TERPSTRA, A.H.M. Influence of amount and type of dietary fat on plasma cholesterol concentrations in goats. Small Ruminant Res., v.35, p.141-147, 2000.

BRINSKO, S.T.; VARNER, D.D.; LOVE, C.C.; BLANCHARD, T.L.; WILSON, M.E. Effect of feeding a DHA-enriched nutriceutical on the quality of fresh, cooled and frozen stallion semen. Theriogenology, v.63, p.1519-1527, 2005.

CARVALHO, G.G.P.; GARCIA, R.; PIRES, A.J.V. et al. Balanço de nitrogênio, concentrações de ureia e síntese de proteína microbiana em caprinos alimentados com dietas contendo cana-de-açúcar tratada com óxido de cálcio. Rev. Bras. Zootec., v.39, p.2253-2261, 2010.

CORDEIRO, M.B.; PERES, M.S.; SOUZA, J.M. et al. Supplementation with sunflower seed increases circulating cholesterol concentrations and potentially impacts on the pregnancy rates in Bos indicus beef cattle. Theriogenology, v.83, p.1461-1468, 2015.

DOLATPANAH; M.B.; TOWHIDI, A.; FARSHAD, A. et al. Effects of dietary fish oil on semen quality of goats. Asian - Aust. J. Anim. Sci., v.21, p.29-34, 2008.

DUNNING, J.; BUTTS, R.; MOURAD, F. et al. Dry needling: a literature review with implications for clinical practice guidelines. Phys. Ther. Rev., v.19, p.252-265, 2014.

ELOY, A.M.X.; PEREIRA, E.P. Estresse na reprodução de caprinos machos. Rev. Bras. Reprod. Anim., v.37, p.156-163, 2013.

FRIEDEWALD, W.T.; LEVY, R.I.; FREDRICKSON, D.S. Estimation of the concentration of low density lipoprotein cholesterol in plasma without use of the preparative ultracentrifuge. Clinic. Chem., v.18, p.499-502, 1972.

GONÇALVES, A.A.; GARCIA, A.R.; ARRUDA, R.P. et al. A suplementação alimentar com ácidos graxos insaturados melhora a qualidade do sêmen criopreservado de búfalos (Bubalus bubalis) pós descongelação. Semin. Ciênc. Agrár., v.35, p.2467-2484, 2014.
GONZÁLEZ, F.H.D.; SILVA, S.C. Introdução à bioquímica clínica veterinária. 2.ed. Porto Alegre: UFGRS, 2006. [30p.].

HENRY, M.; NEVES, J.P.; JOBIM M.I. Manual para exame andrológico e avaliação de sêmen animal. 3.ed. Belo Horizonte: CBRA, 2013. $104 p$.

HUANG, Y.; SCHOONMAKER, J.P.; OREN, S.L. et al. Calcium salts of CLA improve availability of dietary CLA. Livest. Sci., v.122, p.1-7, 2009.

LOTTENBERG A.M.P. Importância da gordura alimentar na prevenção e no controle de distúrbios metabólicos e da doença cardiovascular. Arq. Bras. Endocrinol. Metab., v.53, p.595-607, 2009.

MOALLEM, U.; NETA, N.; ZERONC, Y. Dietary $\alpha$-linolenic acid from flaxseed oil or eicosapentaenoic and docosahexaenoic acids from fish oil differentially alter fatty acid composition and characteristics of fresh and frozen-thawed bull semen. Theriogenology, v.83, p.1110-1120, 2015.

MORAES, G.V.; FÜCK, E.J.; GOMES, M.A.B. et al. Desenvolvimento corporal, avaliações de carcaça e lipedimia de caprinos machos alimentados com grão de linhaça na dieta. Pubvet, v.8, p.1136-1282, 2014.

NUTRIENT requeriments of small ruminants: sheep, goats, cervids, and new world camelids. 5.ed. Washington: National Academy Press, 2007, 384p.

OFFICIAL methods of analysis. 15.ed. Washington: AOAC, 1990.

PAULA, E.F.E.; MAIA, F.P.; CHEN, R.F.F. Óleos vegetais na nutrição de ruminantes. Rev. Eletrôn. Nutritime, v.9, p.2075-2103, 2012.

SAMADIAN, F.; TOWHIDI, A.; REZAYAZDI, K.; BAHREINI, M. Effects of dietary n-3 fatty acids on characteristics and lipid composition of ovine sperm. Anim. Consortium, v.4, p.20172022, 2010.

SNIFFEN, C.J.; CONNOR, J.D.; VAN SOEST, P.J. et al. A net carbohydrate and protein system for evaluation of cattle diets. II Carbohydrate and protein availability. J. Anim. Sci., v.70, p.35623577, 1992. 
SONCIN, M.R.S.P.; FURTADO, C.E.; SILVA, A.A. et al. Digestibilidade aparente, crescimento folicular e concentração de metabólitos sanguíneos de éguas recebendo concentrado com semente de linhaça integral (Linum usitatissimum L.). Acta Sci. Anim. Sci., v.31, p.191-197, 2009.

STAPLES, C.R.; SANTOS, J.E.P.; THATCHER, W.W. Aumento da taxa de prenhez em vacas leiteiras através da suplementação com gordura. In: CURSO NOVOS ENFOQUES NA PRODUÇÃO E REPRODUÇÃO DE BOVINOS, 13., 2009, Uberlândia. Anais... Uberlândia: [BEEF], 2009. p.91-102. (Resumo).
TALEBI, J. Characteristics and seasonal variation in the semen of Markhoz bucks in western Iran. Small Ruminant Res., v.85, p.1822, 2009.

TODINI, L.; MALFATTI, A.; TERZANO, G.M et al. Seasonality of plasma testosterone in males of four Mediterranean goat breeds and in three different climatic conditions. Theriogenology, v.67, p.627-631, 2007.

VAN SOEST, P.J.; ROBERTSON, J.B.; LEWIS, B.A. Methods for dietary fiber, neutral detergent fiber, and nonstarch polyssacharides in relation to animal nutrition. J. Dairy Sci., v.74, p.35833597, 1991. 